\title{
$\gamma$ 谱法测定岩石中的钾含量
}

\author{
夏 明 马志邦 张承德 \\ (中国科学院地质研究所, 北京)
}

$r$ 谱法测定岩石中钾含量, 应该说是比较成熟的一种方法. 早在 1956 年外国学者 ${ }^{[1,2]}$ 就 研究过 $r$ 谱法同时测定不破坏样品中铀、针、铎含量. 在国内应用 $r$ 谱法测定过岩石样品中 铀、镭和针 ${ }^{[3,4,5]}$, 测钾含量甚少,一个主要原因是缺乏相应含钾粉末标准源.

本文配制了一套不同钾含量的粉末源, 确定了 $r$ 谱法中 ${ }^{40} \mathrm{~K}$ 的系数, 测定了各类岩石中的 钾含量, 并取得与化学方法近于一致的结果.

\section{一、方法和实验}

${ }^{40} \mathrm{~K}$ 在钾的总量中占 $0.0118 \%$, 半衰期为 $1.28 \times 10^{9}$ 年, 主要是 $\beta$ 衰变. $\gamma$ 射线的能量为 $1.46 \mathrm{MeV}$, 可以通过这个特征能量光电峰的总计数, 按下式求出样品中钾的含量:

$$
g_{k} / P=a_{k 1} \mathrm{U}+a_{k 2} \mathrm{Th}+a_{k 3} \mathrm{~K},
$$

式中 $g_{k}$ 为 ${ }^{40} \mathrm{~K}$ 光电峰的总计数率; $P$ 一一样品的重量; $a_{k 1} 、 a_{k 2}$ 和 $a_{k 3}$ 分别为单位含量的铀、钍 和钾在 $1.46 \mathrm{MeV}$ 峰面积下总计数中的贡献. 如果样品中铀和铀含量极低, 那么公式 (1) 就可 简化为

$$
g_{k} / P=a_{k 3} \mathrm{~K} .
$$

也就是说, 为了测定钾含量, 首先要确定公式 (2) 中的系数 $a_{k 3}$, 而 $a_{k 3}$ 则要通过与已知标准源 的比较才能确定.

\begin{tabular}{|c|c|c|c|c|c|}
\hline 绿 & 材 料 & 配制诔含量 & $\begin{array}{l}\text { 平均有效原 } \\
\text { 子数数 }\end{array}$ & 平均比重 & $\begin{array}{c}\text { 化学方法测钾含晨 } \\
\text { \% }\end{array}$ \\
\hline $\mathrm{K}-1$ & $\mathrm{KCl}+\mathrm{SiO}_{2}$ & 1.0006 & 11.51 & 2.25 & 1.22 \\
\hline $\mathrm{K}-2$ & $\mathrm{~K}_{2} \mathrm{SO}_{4}+\mathrm{SiO}_{2}$ & 1.0001 & 11.44 & 2.27 & 1.00 \\
\hline $\mathrm{K}-3$ & $\mathrm{~K}_{2} \mathrm{Cr}_{2} \mathrm{O}_{7}+\mathrm{SiO}_{2}$ & 1.0005 & 11.70 & 2.28 & 1.24 \\
\hline $\mathrm{K}-4$ & $\mathrm{KCl}+\mathrm{SiO}_{2}$ & 3.0011 & 11.86 & 2.24 & 3.05 \\
\hline $\mathrm{K}-5$ & $\mathrm{~K}_{2} \mathrm{SO}_{4}+\mathrm{SiO}_{2}$ & 5.0014 & 11.89 & 2.31 & 4.88 \\
\hline $\mathbf{K}-6$ & $\mathrm{KCl}+\mathrm{SiO}_{2}$ & 7.0005 & 12.52 & 2.22 & 7.05 \\
\hline $\mathrm{K}-7$ & $\mathrm{KCl}+\mathrm{SiO}_{2}$ & 11.0008 & 13.14 & 2.20 & 11.41 \\
\hline $\mathrm{K}-8$ & $\mathrm{~K}_{2} \mathrm{SO}_{4}+\mathrm{S}^{\prime} \mathrm{O}_{2}$ & 11.0006 & 12.51 & 2.36 & 10.70 \\
\hline $\mathrm{K}-10$ & $\mathrm{KCl}+\mathrm{SiO}_{2}$ & 15.0002 & 13.72 & 2.18 & 14.94 \\
\hline
\end{tabular}

表 1 含钾粉末源配制含量和参数

* 平均有效原子序数的计算公式为 $\left(\sum_{(2)} P_{x} Z^{2.3}\right)^{1 / 2.3}$.

含钾粉末源是用优级钾盐试剂和二氧化硅做基质配制成的 (表 1). 重量 500 克, 长时间 本文 1984 年 4 月 19 日收到. 
的研磨使钾盐在样品中达到均匀的分布.

r谱仪是由国产 FH-451 型 1024 道分析器和法制 Scintibloc 76SEF76 闪炼低本底组合探 头组成. $\mathrm{NaJ}(\mathrm{TI})$ 晶体为 $3^{\prime \prime} \times 3^{\prime \prime},{ }^{137} \mathrm{Cs}$ 能量分辨率为 $7 \%$. 探头置于大空腔、10 厘米曆的 铅室内. ${ }^{40} \mathrm{~K}$ 的 $1.46 \mathrm{MeV}$ 光电峰选取 50 道, 相当 $135 \mathrm{KeV}$ 能量范围. 在这个区间的空白本底 没有显示出 ${ }^{40} \mathrm{~K}$ 的光电峰, 本底约 210 计数/小时, 或 3.5 计数/分(不带有机玻璃测量盒).

\section{二、结 果}

用 $\gamma$ 谱仪测试的不同重量、不同含钾量的粉末源的数据、基质 $\mathrm{SiO}_{2}$ 粉砂的底数加本底的和 及 $a_{k 3}$ 的平均值列在表 2 . 不同含钾量与 $r$ 计数率的关系以及与化学方法测试结果的对比表示 于图 1 和 2 .

表 2 配制钾粉末源的数据

\begin{tabular}{|c|c|c|c|c|c|}
\hline $\begin{array}{l}\text { 制沫源的重量 } \\
\text { (克) }\end{array}$ & 编 号 & $g_{k}$ 计数/时 & $\mathrm{SiO}_{2}+$ 本底数 & 计数/时 $\times 1 \% \mathrm{~K}$ & $a_{k_{2}}$ 平均值 \\
\hline 300 & $\begin{array}{l}\mathrm{K}-1 \\
\mathrm{~K}-2 \\
\mathrm{~K}-3 \\
\mathrm{~K}-4 \\
\mathrm{~K}-5 \\
\mathrm{~K}-6 \\
\mathrm{~K}-7 \\
\mathrm{~K}-8 \\
\mathrm{~K}-10\end{array}$ & $\begin{array}{r}1388.70 \\
1406.14 \\
1375.76 \\
3575.61 \\
5971.65 \\
8025.90 \\
12651.30 \\
12609.90 \\
17108.33\end{array}$ & $\begin{array}{l}254 \\
254 \\
254 \\
254 \\
254 \\
254 \\
254 \\
254 \\
254\end{array}$ & $\begin{array}{l}1134.02 \\
1152.02 \\
1121.20 \\
1106.78 \\
1143.21 \\
1110.19 \\
1126.95 \\
1123.20 \\
1123.60\end{array}$ & $1126.80 \pm 14.53$ \\
\hline 350 & $\begin{array}{l}\mathrm{K}-1 \\
\mathrm{~K}-2 \\
\mathrm{~K}-3 \\
\mathrm{~K}-4 \\
\mathrm{~K}-5 \\
\mathrm{~K}-6 \\
\mathrm{~K}-7 \\
\mathrm{~K}-8 \\
\mathrm{~K}-10\end{array}$ & $\begin{array}{r}1523.04 \\
1591.32 \\
1516.05 \\
3910.80 \\
6535.80 \\
8960.40 \\
14011.70 \\
14220.90 \\
19009.08\end{array}$ & $\begin{array}{l}254 \\
254 \\
254 \\
254 \\
254 \\
254 \\
254 \\
254 \\
254\end{array}$ & $\begin{array}{l}1268.28 \\
1337.19 \\
1261.42 \\
1218.49 \\
1256.01 \\
1243.68 \\
1250.61 \\
1269.65 \\
1250.32\end{array}$ & $1261.74 \pm 32.18$ \\
\hline 500 & $\begin{array}{l}\mathrm{K}-1 \\
\mathrm{~K}-2 \\
\mathrm{~K}-3 \\
\mathrm{~K}-4 \\
\mathrm{~K}-5 \\
\mathrm{~K}-6 \\
\mathrm{~K}-7 \\
\mathrm{~K}-8 \\
\mathrm{~K}-10\end{array}$ & $\begin{array}{r}1832.55 \\
1867.50 \\
1881.18 \\
4964.70 \\
7992.24 \\
10853.46 \\
17102.30 \\
16906.50 \\
23087.25\end{array}$ & $\begin{array}{l}256 \\
256 \\
256 \\
256 \\
255 \\
255 \\
255 \\
255 \\
255\end{array}$ & $\begin{array}{l}1575.60 \\
1611.34 \\
1624.37 \\
1568.99 \\
1547.01 \\
1513.96 \\
1531.46 \\
1513.69 \\
1522.13\end{array}$ & $1556.50 \pm 41.35$ \\
\hline
\end{tabular}

从图 1 可以看出，在统计涨落内(一般总计数在 10000-100000 之内),配制的不同钾含量 和 $g_{k}$ 之间存在着良好的线性关系, 说明铎在粉末样品中混合均匀. 表 2 的 $a_{k 3}$ 平均值和均方 误差表明, $a_{k 3}$ 测试相对误差低于 $3 \%$ 。

尽管化学方法测钾有较大的波动（见表 1)，但平均值与配制钾含量之间 $45^{\circ}$ 的线性关系 


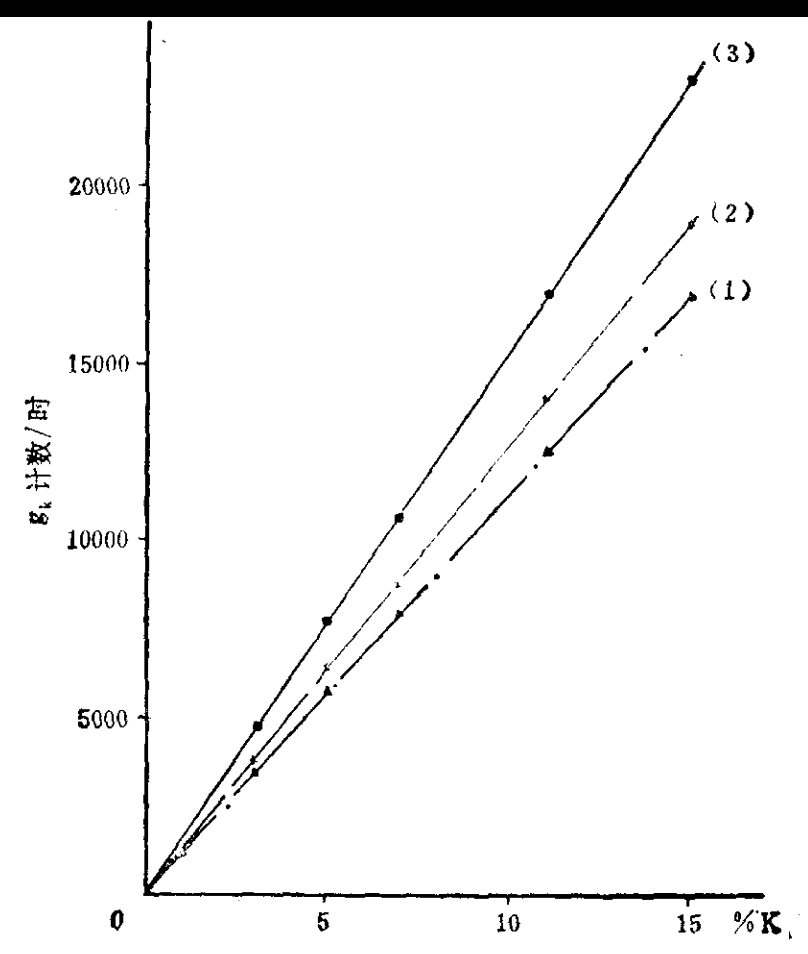

图 1 配制的不同含钾量与计数率的关系 (1) 样品重量 300 克; (2) 样品重量 350 克; (3) 样品重量 500 克

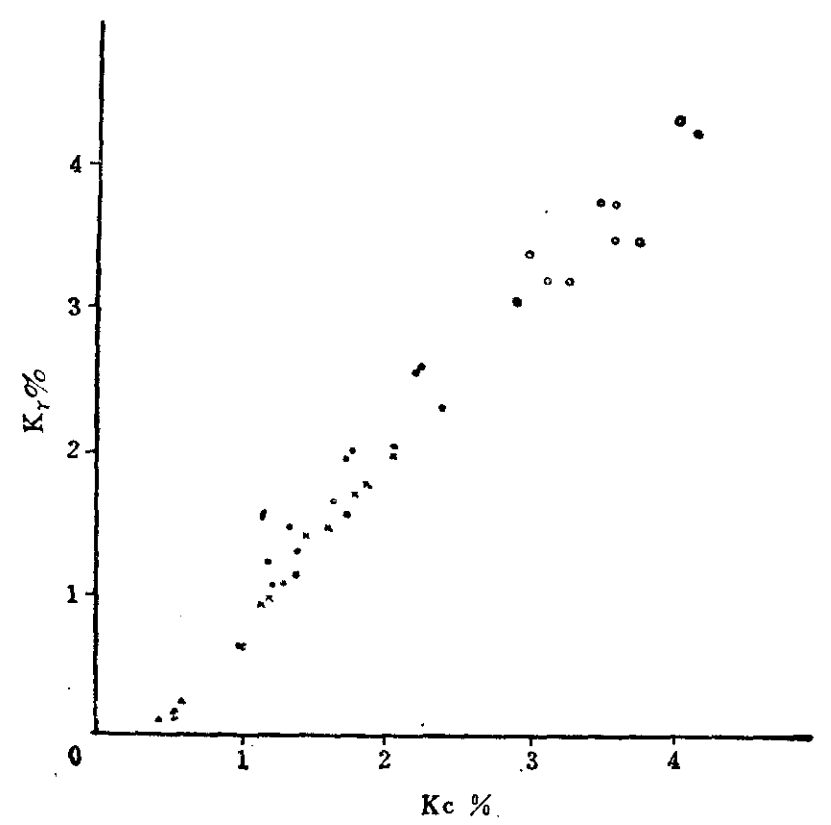

图 $3 r$ 谱法测钾 $\left(K_{r}\right)$ 与化学方法 $\left(K_{c}\right)$ 的比较

○花岗片麻岩; ・角内片麻岩; $\times$ 泥岩; $\Delta$ 龙岩

(见图 2) 反映出不存在系统误差.

应用 $a_{k 3}$ 系数和公式 (1), 测试和计算了近 40 个各种类型岩石中的钾含量,同时也进行了 化学分析, 两种方法的比较见图 3.

两种方法的相关系数为 0.99 , 相关方程为 $K_{r}=1.15 K_{c}-0.35$. 
从灰岩、泥岩、片麻岩到花岗岩, 铸含量零逐步增长的趋势.

测钾 $r$ 能量区间的本底为 220 计数 $/$ 小时, 相应 $<0.2 \%$ 钾含量. 也就是说，近于这个数 值, $r$ 谱法测钾的精度下降 (图 3).

\section{三、结 语}

本实验配制了测定岩石中钾含量的粉末源, 从本身的测试和与化学方法对比来看, 数据是 可靠的, 可以做为实验室级的标准源.

应用 $r$ 谱法测定各种岩石标本中的钾含量,除较低钾含量外,其他与化学测试结果近于一 致.

由于有了钾粉末源，那么应用 $r$ 谱法可以同时测定不破坏岩石样品中的铀、钍和钾含量.

\section{参考文献}

[1] Hurey, P.M., Bull. G.S.A., 67(1956), 4: 395-412.

[2] Якубович А.Л. и Зайцев Е.И., Разведка и Охрана Недр., 33(1961), 2: 33-37.

[3] 夏明, 原子能科学技术, $1965,5: 447-456$.

[4] 基朋等,科学通报, $1965,3: 259-261$.

[5] 曹传虂, 放射性地质, 28(1983)，6: 87-90. 